\title{
Dismantling Limb-Girdle Muscular Dystrophy
}

\section{Citation}

Narayanaswami, Pushpa. 2015. “Dismantling Limb-Girdle Muscular Dystrophy." JAMA

Neurology 72 (12) (December 1): 1409. doi:10.1001/jamaneurol.2015.2749.

\section{Published Version}

doi:10.1001/jamaneurol.2015.2749

\section{Permanent link}

http://nrs.harvard.edu/urn-3:HUL.InstRepos:33973672

\section{Terms of Use}

This article was downloaded from Harvard University's DASH repository, and is made available under the terms and conditions applicable to Other Posted Material, as set forth at http:// nrs.harvard.edu/urn-3:HUL.InstRepos:dash.current.terms-of-use\#LAA

\section{Share Your Story}

The Harvard community has made this article openly available.

Please share how this access benefits you. Submit a story.

Accessibility 


\section{Dismantling Limb-Girdle Muscular Dystrophy The Role of Whole-Exome Sequencing}

Pushpa Narayanaswami, MBBS, DM

Muscular dystrophy encompasses a diverse group of genetically determined muscle disorders. The first clinical description of the disorder is attributed to Giovanni Semmola, who, in 1829, described 2 boys affected by a disorder with prominent muscular hypertrophy. ${ }^{1}$ $\leftarrow$ Between 1850 and 1868, Aran, Related article page 1424 Meryon, and Duchenne described a progressive atrophy of voluntary muscles, ultimately termed pseudohypertrophic muscular paralysis of children by Duchenne. ${ }^{1,2}$ Other descriptions followed: familial atrophy of the pelvic girdle muscles (Leyden in 1876), scapulohumeral muscular atrophy (Erb in 1884), and myopathy with facial weakness (Landouzy and Dejerine in 1884). ${ }^{1}$ The term limb-girdle muscular dystrophy (LGMD), suggested by Stevenson in $1953,{ }^{3}$ and further detailed by Walton and Nattrass in a seminal article, ${ }^{2}$ refers to a group of muscular dystrophies with onset of weakness in the shoulder or pelvic girdles. ${ }^{4}$ The variable clinical course of this disorder was recognized even in these early descriptions. ${ }^{2,3}$

Traditional neurology emphasizes the role of the history and clinical examination in arriving at a diagnosis. The clinical approach to patients with suspected myopathy includes obtaining a history of symptom evolution, comprehensive pedigree analysis, evaluation of the distribution of weakness, and identification of extramuscular manifestations. Neuromuscular neurologists often use a "pattern recognition" approach, which classifies muscle weakness into specific schema: limb girdle, distal, humeroperoneal, etc. There is value to this clinical approach, which allows one to narrow the diagnosis down to a few conditions, or even a single condition, to inform subsequent confirmatory testing. A young boy with onset of weakness in the first decade, calf hypertrophy, Gowers maneuver, and a similarly affected maternal uncle is likely to have Duchenne muscular dystrophy. For a young adult with myotonia, ptosis, temporalis atrophy, distal limb weakness, and a family history suggesting autosomal dominant inheritance, clinical suspicion of myotonic dystrophy leads to confirmatory genetic testing. However, genetic heterogeneity (ie, the existence of similar phenotypes due to different genetic mutations) limits the diagnostic capacity of phenotypic classifications.

In 1987, the discovery of the genetic defect and protein product, dystrophin, in Duchenne muscular dystrophy revolutionized the approach to muscular dystrophies. ${ }^{5}$ An era of molecular diagnoses was born. As the search for genetic causes for neuromuscular disorders continued, it became apparent that LGMD was not a single disease but likely represented several disorders unified by their phenotype. After reports of autoso- mal recessive LGMD from the 1950s on, Fardeau and colleagues, in 1989, described an autosomal recessive LGMD on the French island of Réunion; in 1991, this disorder was found to link to chromosome 15q, and in 1995, it was identified as calpainopathy. ${ }^{6}$ In 1986, a report of autosomal dominant LGMD established the genetic heterogeneity of this syndrome. ${ }^{7}$ A subsequent family with autosomal dominant LGMD reported in 1988 was found to have a type of LGMD that linked to chromosome 5q in 1992, and in 2000, this family was identified as having a mutation in the myotilin gene. ${ }^{8}$ A genetic classification of LGMDs was proposed in 1995, based on the inheritance pattern: Type 1 LGMDs are autosomal dominant, and type 2 LMGMDs are autosomal recessive. A letter defining the order of discovery of the chromosomal locus is appended to the numeric designation. ${ }^{9}$ The list of LGMDs with known genetic loci continues to grow rapidly (8 autosomal dominant LGMDs [LGMD1A$1 \mathrm{H})$ and 23 autosomal recessive LGMDs [LGMD2A-2W]). ${ }^{10}$

A definitive diagnosis of the type of LGMD is important; it avoids repeated testing or empirical, potentially toxic treatments for acquired causes such as inflammatory myopathy; provides patients with a sense of closure; assists genetic counseling; and aids the identification and treatment of complications. ${ }^{11}$ In the future, knowledge of the underlying genetic mutation will be necessary for enrollment in clinical trials of targeted therapies. However, the genetic heterogeneity of the LGMD phenotype makes establishing a definitive diagnosis challenging. The approach to diagnosis is complex and includes evaluation of the inheritance pattern and identification of specific clinical features that direct further testing. ${ }^{11}$

Conventional genetic testing for mendelian disorders such as LGMD involve genome-wide linkage to identify mutations that cosegregate within affected individuals, positional cloning, and, finally, targeted candidate gene sequencing. ${ }^{12}$ In autosomal recessive disorders, autozygosity mapping, which identifies regions of the genome that are homozygous in affected individuals but not in unaffected family members, is followed by gene sequencing to identify the causal mutation..$^{13}$ These techniques assume the availability of several affected family members and are of limited utility when only a few cases are available or in sporadic cases owing to de novo mutations. In addition, genetic and phenotypic heterogeneity and incomplete penetrance limit these approaches. ${ }^{13}$

The DNA sequencing technique described by Sanger et $\mathrm{al}^{14}$ was used to sequence the human genome in 2001 (the Human Genome Project). ${ }^{15}$ In 2004, next-generation sequencing methods (which use high-throughput, massively parallel sequencing platforms) were introduced, making it possible to sequence sev- 
eral thousand or more fragments of DNA simultaneously. Nextgeneration sequencing platforms can complete genome sequencing within a few weeks at a fraction of the cost of Sanger sequencing. In addition to whole-genome sequencing, next-generation sequencing can be used for whole-exome sequencing (WES). The exome is the sequence of all exons of protein-coding genes, covering $1 \%$ to $2 \%$ of the genome, and contains an estimated $85 \%$ of the mutations that cause mendelian disorders. ${ }^{16}$ Hence, WES, or targeted sequencing of all known exons, is often sufficient to identify the genes underlying mendelian disorders.

In WES, genomic DNA is first extracted and broken down into short segments. Priming sequences of DNA are added to the ends of these fragments. The fragments are then captured by mixing them with probes that are complementary to known exons in the genome. The remaining DNA is washed away, leaving the enriched exome sequences for amplification and massive parallel sequencing. Fluorescent nucleotides incorporated into strands of DNA during the sequencing process are detected using laser activation. Millions of short sequence reads are mapped to a reference genome, and variants within the genome are identified. ${ }^{12,17}$ These variants are then filtered to exclude nonpathogenic polymorphisms. The first step is to crossreference the variants against publicly available databases of exomes from unaffected individuals. The remaining variations are then filtered by the mode of inheritance, segregation within affected family members, stratification, etc. ${ }^{17}$

In this issue of JAMA Neurology, Ghaoui and colleagues ${ }^{18}$ describe the application of WES to evaluate patients whose LGMD subtype remained undiagnosed after immunohistochemistry of muscle biopsy specimens and targeted Sanger sequencing, which provided a diagnosis for 83 of 237 families (35\%). Of the remaining 154 families, 100 individuals from 60 families underwent WES. Ghaoui et al ${ }^{18}$ identified pathogenic mutations in 27 of 60 families (45\%). Importantly, they studied 45 probands without parental DNA, in whom the diagnostic rate of WES was a respectable $40 \%$, underscoring the value of this technique in sporadic cases. Twelve families had a known LGMD-related gene mutation. Six of these represented previously reported mutations; it is unclear why these were missed by candidate gene testing.

More interestingly, 14 of 60 families had mutations in genes that are not classically associated with an LGMD phenotype (GTDC2, LAMA2, and ACTA1), congenital myasthenia due to DOK7 mutation, or even metabolic myopathy (PYGM and CPT2). A second look at the phenotype helped confirm the diagnosis in several cases: clinical features missed at initial examination (contractures, scoliosis, follicular hyperkeratosis and keloids in Bethlem myopathy, low-set ears in STIM1-related myopathy with tubular aggregates, and fatigability in congenital myasthenia), review of muscle biopsy (reduced collagen staining, reduced protein detected on immunohistochemistry and Western blot, and absence of enzyme detected on histochemistry), magnetic resonance imaging of the limbs for patterns of muscle atrophy or magnetic resonance imaging of the brain for white matter disease. In 1 family, a novel heterozygous variant in the $C H D 7$ gene was detected. The proband had proximal upper extremity weakness and scapular winging with mild myopathy detetected in biopsy specimen. Other phenotypic features of CHARGE syndrome (coloboma, heart defect, atresia of the choanae, retarded growth, and genital abnormalities) were not present, but low-set ears and magnetic resonance imaging evidence of hypoplasia of the semicircular canals were noted (ear abnormalities).

This study ${ }^{18}$ supports the utility of WES for the genetic diagnosis of LGMD. However, important ethical considerations include informed consent and the management of clinically important, unrelated genetic information that may be incidentally uncovered. Ghaoui et $\mathrm{al}^{18}$ correctly point out the limitations of WES; incomplete enrichment or "coverage" of the sequences of interest is one limitation. As a consequence, a sequencing error may be falsely interpreted as a mutation, or a true mutation may be missed. Similarly, slight misalignment of the sequenced DNA to the reference genome may cause small base substitutions or insertions/deletions to be missed, or create the impression of new mutations. ${ }^{17}$ Because the DNA probes are based on sequences from known exons, mutations in unknown exons will be missed. Pathogenic mutations in noncoding elements, introns, regulatory elements such as promoters and enhancers, or splice site mutations will also not be detected. ${ }^{16}$ Repeat expansions are generally not detected with exome sequencing, and Ghaoui et $\mathrm{al}^{18}$ appropriately excluded type 2 myotonic dystrophy and type $1 \mathrm{fa}$ cioscapulohumeral muscular dystrophy prior to WES in their study. Finally, filtering techniques may erroneously omit pathogenic mutations because mutation carriers who have not yet expressed the disease owing to reduced penetrance may be present in the control databases. ${ }^{17}$

Where, then, does the clinical armamentarium of the traditional neurologist fit in? Is it time to replace the phenotypic classification with a genetic classification of muscular dystrophies? A recent report of the National Academy of Sciences recommends a new, dynamic taxonomy of human diseases incorporating molecular biology, clinical data, and environmental data to improve health care. ${ }^{19}$ The influence of yet-to-be discovered epigenetic factors on disease expression must also be considered. The genetic diagnosis of muscular dystrophies is rapidly evolving; the jury is still out on the optimal diagnostic algorithms and techniques and their clinical and genetic implications. Close attention to clinical examination incorporating not only the neurologic examination but also a detailed multisystem examination remains an essential part of the diagnostic algorithm in muscular dystrophy. Long-term follow-up will provide valuable phenotypegenotype correlations for management of future patients. It is not yet time to retire our reflex hammers.

\section{ARTICLE INFORMATION}

Author Affiliation: Department of Neurology, Beth Israel Deaconess Medical Center/Harvard Medical School, Boston, Massachusetts.

Corresponding Author: Pushpa Narayanaswami, MBBS, DM, Department of Neurology, Beth Israel
Deaconess Medical Center/Harvard Medical School, Neurology TCC-8, 330 Brookline Ave, Boston, MA 02215 (pnarayan@bidmc.harvard.edu).

Published Online: October 5, 2015. doi:10.1001/jamaneurol.2015.2749.

Conflict of Interest Disclosures: None reported.
Additional Contributions: I would like to thank S. Rutkove, MD, at the Department of Neurology, Beth Israel Deaconess Medical Center, Boston, Massachusetts, for reviewing the manuscript and making suggestions on the content. He did not receive financial compensation. 


\section{REFERENCES}

1. Angelini C. Chapter 31: muscular dystrophy. Handb Clin Neurol. 2010;95:477-488.

2. Walton JN, Nattrass FJ. On the classification, natural history and treatment of the myopathies. Brain. 1954;77(2):169-231.

3. Stevenson AC. Muscular dystrophy in Northern Ireland, I: an account of the condition in fifty-one families. Ann Eugen. 1953;18(1):50-93; contd.

4. Bushby KM. Diagnostic criteria for the limb-girdle muscular dystrophies: report of the ENMC Consortium on Limb-Girdle Dystrophies. Neuromuscul Disord. 1995;5(1):71-74.

5. Koenig M, Hoffman EP, Bertelson CJ, Monaco AP Feener C, Kunkel LM. Complete cloning of the Duchenne muscular dystrophy (DMD) CDNA and preliminary genomic organization of the DMD gene in normal and affected individuals. Cell. 1987;50 (3):509-517.

6. Richard I, Broux O, Allamand V, et al. Mutations in the proteolytic enzyme calpain 3 cause limb-girdle muscular dystrophy type 2A. Cell. 1995; 81(1):27-40.

7. Chutkow JG, Heffner RR Jr, Kramer AA, Edwards JA. Adult-onset autosomal dominant limb-girdle muscular dystrophy. Ann Neurol. 1986; 20(2):240-248.
8. Hauser MA, Horrigan SK, Salmikangas $P$, et al. Myotilin is mutated in limb girdle muscular dystrophy 1A. Hum Mol Genet. 2000;9(14):2141-2147.

9. Bushby KM, Beckmann JS. The limb-girdle muscular dystrophies-proposal for a new nomenclature. Neuromuscul Disord. 1995;5(4): 337-343.

10. Nigro V, Savarese M. Genetic basis of limb-girdle muscular dystrophies: the 2014 update. Acta Myol. 2014;33(1):1-12.

11. Narayanaswami $P$, Weiss $M$, Selcen $D$, et al; Guideline Development Subcommittee of the American Academy of Neurology; Practice Issues Review Panel of the American Association of Neuromuscular \& Electrodiagnostic Medicine. Evidence-based guideline summary: diagnosis and treatment of limb-girdle and distal dystrophies: report of the guideline development subcommittee of the American Academy of Neurology and the practice issues review panel of the American Association of Neuromuscular \& Electrodiagnostic Medicine. Neurology. 2014;83(16):1453-1463.

12. Keogh MJ, Chinnery PF. Next generation sequencing for neurological diseases: new hope or new hype? Clin Neurol Neurosurg. 2013;115(7): 948-953.

13. Rabbani B, Mahdieh N, Hosomichi K, Nakaoka $\mathrm{H}$, Inoue I. Next-generation sequencing: impact of exome sequencing in characterizing Mendelian disorders. J Hum Genet. 2012;57(10):621-632.
14. Sanger F, Nicklen S, Coulson AR. DNA sequencing with chain-terminating inhibitors. Proc Natl Acad Sci U S A. 1977;74(12):5463-5467.

15. McPherson JD, Marra M, Hillier L, et al; International Human Genome Mapping Consortium. A physical map of the human genome. Nature. 2001;409(6822):934-941.

16. Majewski J, Schwartzentruber J, Lalonde E, Montpetit A, Jabado N. What can exome sequencing do for you? J Med Genet. 2011;48(9): 580-589.

17. Bamshad MJ, Ng SB, Bigham AW, et al. Exome sequencing as a tool for Mendelian disease gene discovery. Nat Rev Genet. 2011;12(11):745-755.

18. Ghaoui R, Cooper ST, Lek M, et al. Use of whole-exome sequencing for diagnosis of limb-girdle muscular dystrophy: outcomes and lessons learned [published online October 5, 2015]. JAMA Neurol. doi:10.1001/jamaneurol.2015.2274.

19. Committee on a Framework for Development a New Taxonomy of Disease; Board of Life Sciences; Division on Earth and Life Studies; National Research Council. Toward Precision Medicine: Building a Knowledge Network for Biomedical Research and a New Taxonomy of Disease. Washington, DC: National Academies Press; 2011.

\section{Equivalence of Glatiramer Acetate Generics With Branded Glatiramer Acetate in Efficacy and Cost for the Treatment of Multiple Sclerosis}

Dennis Bourdette, MD; Daniel Hartung, PharmD, MPH

Between 1993 and 2015, the US Food and Drug Administration (FDA) approved 13 drugs for the treatment of relapsingremitting multiple sclerosis (MS). While these diseasemodifying therapies (DMTs) fall into 7 different categories and vary in route of administra$\leftarrow$ Related article page 1433 tion, adverse effects, and efficacy, they have one commonality-they are all very expensive, with acquisition costs exceeding $\$ 60000$ a year and continuing to escalate. ${ }^{1}$ One potential reason for the skyrocketing costs of MS DMTs is that they have never faced price competition from lower-cost generic drugs. The recent FDA approval of a generic version of branded glatiramer acetate (Copaxone; Teva Pharmaceuticals) signals the promise of true price competition among MS DMTs. However, the economic effect of the availability of a generic DMT is as yet unclear because this depends on the price of the generic and the extent of its use. Although pricing decisions are beyond our control, neurologists can have a large influence on how generic DMTs are used. As such, neurologists need to assess whether or not generic glatiramer acetate is equivalent to branded glatiramer acetate and acceptable for use in treating MS.
Glatiramer acetate emerged from research started in the 1960s at the Weizmann Institute of Science. Arnon and colleagues $^{2,3}$ assessed the effects of synthetic polypeptides on the MS model of experimental autoimmune encephalomyelitis. The glatiramer acetate products contain polypeptides formed from the random polymerization of L-alanine, L-lysine, L-glutamic acid, and L-tyrosine in a molar ratio of 6.0: 4.7:1.9:1. Glatiramer acetate contains polypeptides of varying lengths that fall within a defined molecular weight range (4.7-10 $\mathrm{kDa})$. Therefore, it is a complex drug and not a simple smallmolecule drug. The first successful double-blind placebocontrolled trial of glatiramer acetate in relapsing-remitting MS used the drug made in small batches at the Weizmann Institute of Science. ${ }^{2,4}$ The development of the manufacturing techniques and quality controls needed to produce a commercial product took Teva Pharmaceuticals about 2 years and was not a trivial process. ${ }^{2}$ It was this version of glatiramer acetate that was studied in the pivotal phase 3 trial and eventually be-

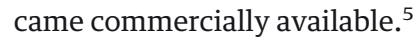

In 1995, the FDA approved branded glatiramer acetate, injected subcutaneously in a dose of $20 \mathrm{mg} / \mathrm{d}$, and it is now widely prescribed. Glatiramer acetate has an outstanding rec- 